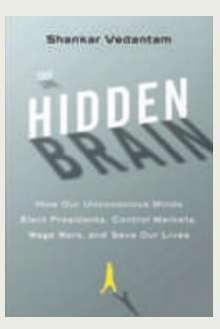

Some of our biggest decisions are made without conscious awareness, argues Washington Post columnist Shankar

Vedantam in The Hidden Brain (Spiegel \& Grau, 2010). Using powerful case studies, Vedantam examines with a light touch unconscious bias and sexism; why we seem to care more about animal welfare than genocide; and how decisions taken by those on the 88th and 89th floors of the World Trade Center's South Tower on 11 September 2001 determined whether they lived or died.

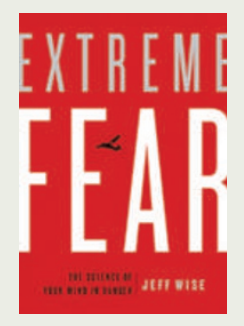

Practising what he preaches, journalist Jeff Wise jumped out of a plane and was fired on by missiles while researching his book Extreme

Fear (Palgrave Macmillan, 2009). Taking a hands-on approach, Wise offers a series of gripping tales to illustrate how our brains function when we are exposed to mortal danger and how we can learn to control anxiety. Using the experiences of people who have averted lion attacks, wildfires and plane crashes, as well as of stage performers and top athletes, Wise explains how to stay cool under pressure.

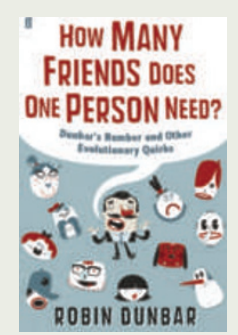

From why we kiss to how religious we are, we can't escape our evolutionary history, explains anthropologist Robin Dunbar in How Many

Friends Does One Person Need? (Faber \& Faber, 2010). With clear descriptions of groundbreaking experiments in evolutionary biology aimed at a popular audience, he explains why all babies are born premature, the science behind lonely hearts columns and why, even in the era of Facebook, we can never be acquainted with more than 150 people.

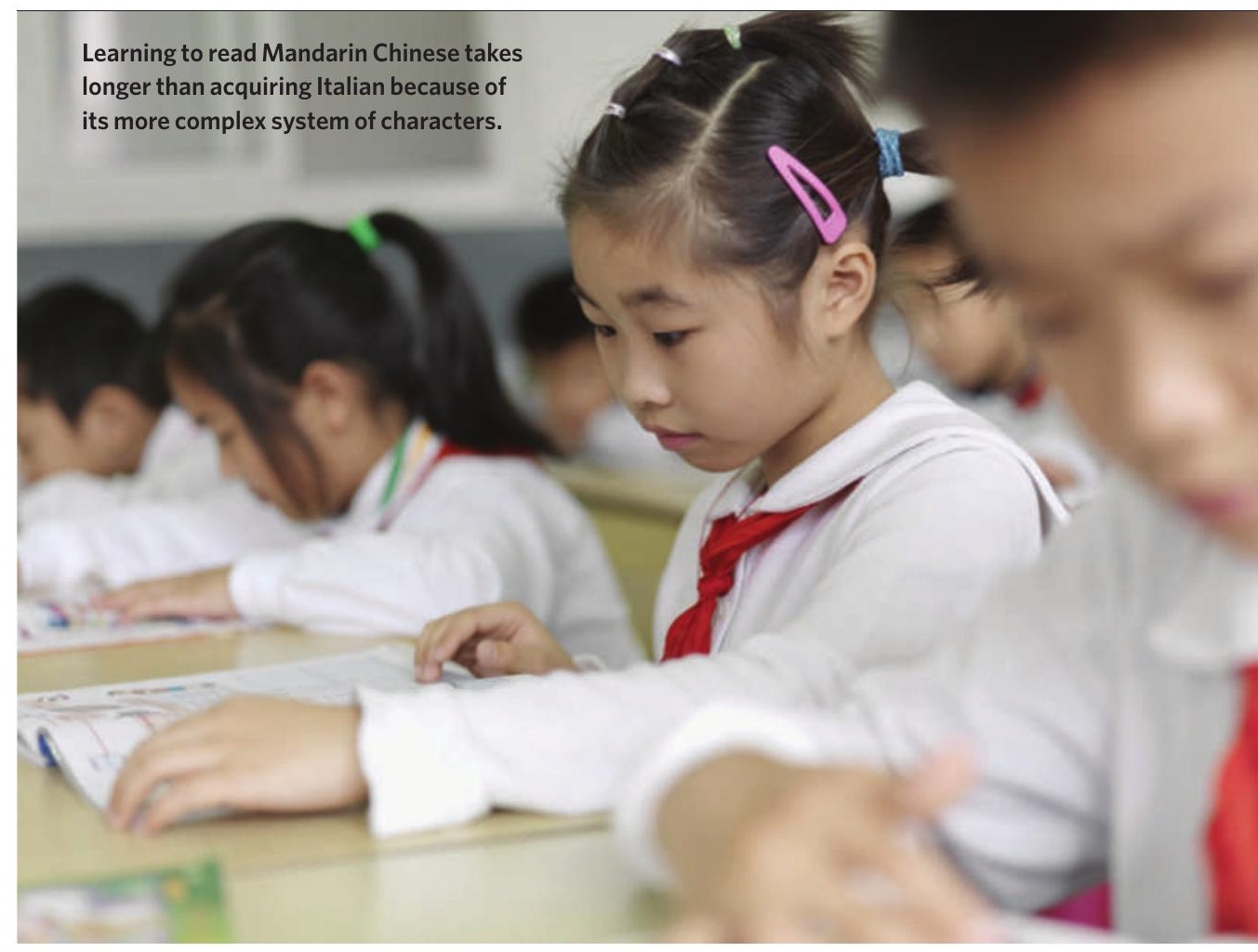

\title{
Deciphering the printed word
}

Reading is a vital portal to knowledge. Unique to humans, this evolutionarily recent invention intertwines language and vision in such a new way that years of education are needed to become fluent. The written word occurs in a dazzling variety of writing systems, from Roman and Greek alphabets to Chinese and Japanese characters, reflecting both universal similarities and the idiosyncratic evolutions of different languages. In his accessible and provocative book Reading in the Brain, cognitive neuroscientist Stanislas Dehaene explains how our brain empowers us to read.

A leading researcher in this field, Dehaene views reading as a tour de force of the human brain that is intellectually fascinating and important for education. Understanding how we read requires consideration of many themes: psychology, the organization of vision and language in the brain, primate neurophysiology and its evolution, the history of writing, the development of the child's mind and brain, and cultural variation. The book weaves these aspects into a compelling synthesis.

Dehaene describes pertinent neurological cases, such as that of a man with word blindness. After having a stroke, the man's vision was intact and he could hear, speak and write down dictated words, but he couldn't read words, not even those he had written himself. Dehaene explains that when we read, we visually process about 12 letters at a time. He reveals the neural architecture of reading, describing the role of brain regions such as the 'letterbox', in which printed words are recognized as the gateway to meaning.

A theme throughout the book is how reading involves a balance between evoking the sound of a word in spoken language and rapidly translating print to meaning. The competing auditory and visual demands produce two interactive pathways in the brain - a phonological route that translates printed words to sound and then meaning, and a direct route that translates print to meaning. The reading system is so impressive that we can identify a word, from among the 50,000-100,000 words that we know, in a mere 50 thousandths of a second.

Dehaene then takes us on a global tour. Languages vary markedly in how they are written down and how that print relates to sounds and words. In Italian, letters or small groups of letters - known as graphemes - correspond almost on a one-to-one basis to the smallest 
linguistic units of sound, or phonemes, with about a 33:25 ratio of graphemes to phonemes. By contrast, on account of its complex history, the English language has to ascribe a greater variety of sounds using the same alphabet. Thus it contains many irregularities, such as the evocation of different sounds with the same letter. This results in about a 1,120:40 ratio of graphemes to phonemes. In Mandarin Chinese, thousands of characters reflect the mixture of words and information about pronunciation.

This variation in writing systems has profound effects on education. A child becomes a skilled reader in Italian in about a year, in English in about three years, and in Mandarin Chinese in about a decade. Cultural variation places different demands on brains that share universal principles of organization.

Dehaene is forthright about how scientific evidence about reading can contribute to educational policies and practices, and about the limits of such contributions. He argues that children should be taught 'phonics' as a core component of initial reading - a learning process that connects the sounds of spoken language with individual or groups of letters in alphabetic languages. He also explains how evidence for the contrasting method of 'whole-word' reading was misunderstood in promoting its most extreme versions.

The book ends by exploring two fascinating topics. First, Dehaene considers the remarkable frequency with which children reverse the writing of letters. He argues that this developmental phase may reflect a residual consequence of our specialized ability to recognize print, emerging from our general ability to recognize visual objects. Second, he considers how reading may be one case of a uniquely human brain capacity to create new cultures, thanks to neural systems that support multimodal integration, abstract thoughts and our ability to represent the intentions and beliefs of others - known as the theory of mind. It takes a powerful theory of mind for the writer to imagine what can be communicated to the reader.

Dehaene's masterful book is a delight to read and scientifically precise. It is vibrant with intellectual curiosity, fascinating perspectives and amusing examples. Reading in the Brain is compelling for anyone who is curious about how we read.

John Gabrieli is Grover Hermann Professor in Health Sciences and Technology and Cognitive Neuroscience at the Massachusetts Institute of Technology, Cambridge,

Massachusetts 02139-4307, USA.

e-mail: gabrieli@mit.edu

\section{Across the cultural divide}

\author{
The Neural Imagination: Aesthetic and \\ Neuroscientific Approaches to the Arts \\ by Irving Massey \\ University of Texas Press: 2009. 195 pp. \\ $\$ 55$
}

The history of neuroaesthetics might be described as an epic war that has been fought between scientists and artists since the days of Freudian psychology and surrealism. Both sides - one hopeful, one fearful - posit that science might eventually explain away the arts. In The Neural Imagination, Irving Massey asks how close this fantasy comes to reality, as revealed by academic research.

As emeritus professor of English and comparative literature at the State University of New York at Buffalo, Massey offers a revealing response to earlier works on neuroaesthetics by neurobiologists - notably Semir Zeki's Inner Vision (Oxford University Press, 1999) and Margaret Livingstone's Vision and Art (Abrams, 2002). As well as discussing visual art, Massey considers our responses to music and language.

The deep clash between art and science that is inherent to the neuroaesthetics field is fuelled by a fundamental concern: to what extent is appreciation of art driven by the automatic responses of human physiology and biology, versus the intellectual and emotional deliberations of the culture-influenced observer? Massey highlights extreme viewpoints through provocative scenarios.

He considers, for example, forms of art that are designed to induce purely automatic responses. The act of art appreciation can then be likened to the way a frog continues to twitch when its head is cut off. Such a comparison seems simplistic, but it does reflect the widespread fear of the 'physiology is destiny' approach to art. Equally, the 'culture is destiny' aficionados cannot distance art from physiology. After all, they witnessed the drastic evolution in the painting style of Willem De Kooning as his Alzheimer's disease progressed.

Here Zeki falls within Massey's sights. Zeki is famous for using functional magnetic resonance imaging (fMRI) to catalogue art based on the regions of the observer's brain that are activated, and later proposing that a 'beauty spot' lies in the orbito-frontal cortex. Massey expresses his concern by taking Zeki's vision one step further - if we can identify the region that is activated when looking at, say, a Monet, then might we replace the original artwork with technology that allows us to stimulate this region directly?

Massey considers that the interplay between art, the mind and the brain will be crucial for our future understanding of humanity. He supports scientific exploration, but feels that it can go only so far. Science, he declares, can explain the 'how' but not the 'why' of artistic effects. For example, neuroscience can explain why stationary patterns are perceived to move in some artworks, such as the spinning circles of Isia Leviant's Enigma (1981). But it cannot judge the significance of the motion or its aesthetic value.

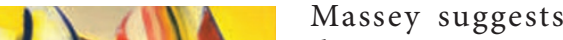
that neuroscientists should collaborate with art theorists to integrate the scientific findings within the contexts of the acts of creation and observation of artwork.

Looking at analogous concerns in music, he says that, although it is possible to understand the role of pitch and tempo for inducing aesthetic sensations such as relaxation or anticipation, the neurology of musical effects remains elusive. For language, where his academic expertise lies, he discusses the future potential of fMRI studies, particularly for investigating dreams because, he explains, "In dreams we speak our language; when awake, the language of others."

Massey does not have all the answers. But in reading his appealing book we are witnessing a researcher thinking out loud. A common enthusiasm for a research field may one day defeat cultural divides, and the human mind and its relationship to the arts is one of the best contenders for collaboration.

Richard Taylor is professor of physics, psychology and art in the Department of Physics at the University of Oregon, Eugene, Oregon 97403, USA.

e-mail:rpt@uoregon.edu 